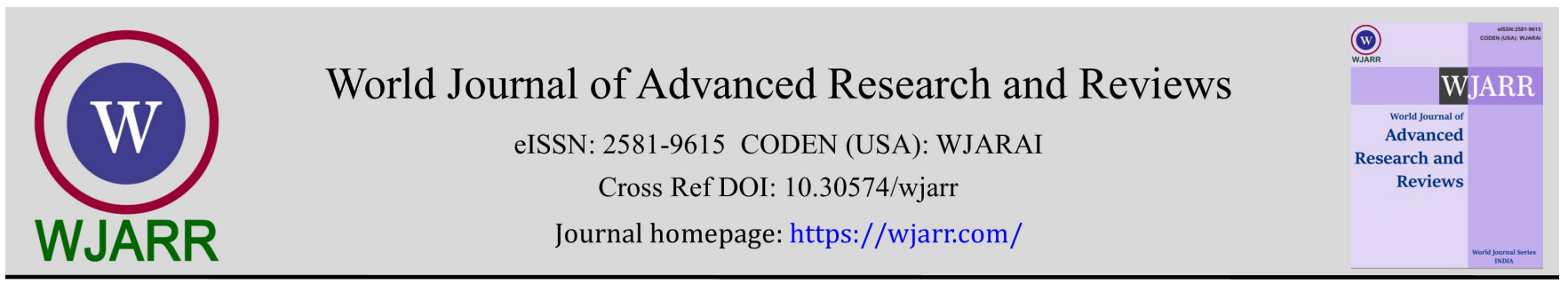

(RESEARCH ARTICLE)

\title{
Tai Chi Chuan improves cytokine expression levels and quality of life in post chemotherapy breast cancer survivors
}

\author{
Mary NB Cheung 1, 2 and Tjing Yung Loo 1, 2, * \\ ${ }^{1}$ Institute for Research and Practice of Yang Style Tai Chi Chuan, Hong Kong, China. \\ ${ }^{2}$ Keenlink Dental Clinic \& Essence Medical Laboratory, Hong Kong, China.
}

World Journal of Advanced Research and Reviews, 2021, 10(03), 322-333

Publication history: Received on 21 April 2021; revised on 06 June 2021; accepted on 10 June 2021

Article DOI: https://doi.org/10.30574/wjarr.2021.10.3.0244

\begin{abstract}
Background: This study investigated the effects of Tai Chi Chuan (TCC) exercise on biological markers and psychological factors of breast carcinoma patients diagnosed with advanced invasive ductal carcinoma.

Methods: Sixty breast cancer patients, and 50 healthy female subjects. Patients underwent a mastectomy for invasive ductal carcinoma, and completed 4 cycles of 5-fluorouracil, epirubicin and cyclophosphamide chemotherapy. Peripheral blood was drawn from subjects to analyze the biological markers, namely white blood cell (WBC) and red blood cell (RBC) count and cytokine levels (IL2, IL4, IL6, IFN- $\gamma$ and TNF- $\alpha$ ). Following treatment, the patients participated in a TCC program for 12 months. Biomarkers and hand grip strength were measured at baseline, 6 and 12 months after TCC practice. A psychological factor-based questionnaire was also conducted.
\end{abstract}

Results: After 12 months of TCC, the levels of cytokines of patients achieved no statistically significant difference compared to healthy controls. The total WBC count was $7.55 \pm 1.25(\mathrm{p}<0.05)$, and the total RBC count was $4.62 \pm 1.25$ $(\mathrm{p}<0.05)$. Patients noticed improvements in short term memory. Following 12 months of TCC practice, patients also had stronger hand grip strength with baseline.

Conclusions: TCC exercise can recommended for the rehabilitation of post-treatment breast cancer patients for biological and psychological improvements.

Keywords: Breast cancer; Tai Chi Chuan; Cytokines; Psychological factors

\section{Introduction}

Tai Chi Chuan (TCC), a well-known traditional Chinese martial art, originated from a Dao philosophical principal that states the cosmos, the earth, and the human are combined together and are balanced by each other to form a system (1) . This entire system will not function if any part is missing or unbalanced. The internal system of the human body also counterbalances with both the outside world and the internal homoeostasis (2) . According to the Dao thought, disruption to any part of the system will lead to the development of diseases (2) .

TCC has long been widely practiced among Chinese communities worldwide for its health benefits and extending life span (3) . TCC is considered a therapy, a preventive measure and a remedy for almost every ailment, including arthritis (4) . Tracing back to ancient China in the $12^{\text {th }}$ century, TCC was first developed as a form of self-defense and was later

\footnotetext{
* Corresponding author: Tjing Yung Loo

Institute for Research and Practice of Yang Style Tai Chi Chuan, Hong Kong, China.

Copyright (@ 2021 Author(s) retain the copyright of this article. This article is published under the terms of the Creative Commons Attribution Liscense 4.0.
} 
classified as a Chinese martial art form (5). It was not until the late 18th century that people slowly found TCC exercises to have health and meditative influences (5) .

TCC is presently widely practiced in different styles, most noticeably for relaxation and as a form of moderate exercise of rhythmic movement, incorporating relaxation and meditation, and focuses strongly on the concentration of mind (6) . TCC is characterized by its flowing, dance-like movements, complete weight shifts, moving from deep relaxation to full speed and force, requiring integration of the mind and body in every movement through respiration, mental and visual concentration (7). The mental requirements of the individual allow this form of art to act as a vehicle for meditation, relaxation and spiritual well-being (8). TCC is classified as body-mind practice (9). Throughout this body-mind practice, the Chinese believe that the person practicing this martial art form could communicate with his/her internal body; hence, this could alter the internal body and recover from diseases.

Breast cancer is the leading cause of female deaths for both developing and developed countries. Diagnosing breast cancer and the subtype will help direct the clinical treatment program, with the primary aims of curing, prolonging the time to disease progression and improving long-term survival, or to ensure the best quality of life for patients in the case of palliative care. Patients diagnosed with breast cancer are faced with different treatment options, of which surgery for removal of the tumour(s) and chemotherapy are common considerations.

Chemotherapy works by eradicating not only cancerous cells in patients, but also rapidly dividing healthy cells in hair follicles that causes loss of hair in patients, as well as suppressing the levels of white blood cells (WBC) and red blood cells (RBC). Chemotherapy has many side effects such as nausea, vomiting, oral ulcerative mucositis, diarrhea, and myelosuppression (10-13) . Oral ulcerative mucositis represents a major non-hematologic complication of cytotoxic chemotherapy and radiotherapy associated with significant morbidity, pain, odynodysphagia, dysgeusia, and subsequent dehydration and malnutrition reduce the quality of life of affected patients. Oral ulcers represent a significant risk factor for systemic infections, particularly in neutropenic patients. There were about $30 \%$ of patients who receive chemotherapy for the treatment of cancer (10-13) accompany with ulcerative lesions, the mucositis produced by stomato-toxic chemotherapy are painful, restrict oral intake and, importantly, act as sites of secondary infection and portals of entry for the endogenous oral flora (13).

The traumatic experience of facing a major physical change for breast cancer patients will, without a doubt, cause posttraumatic stress disorder and psychological problems. The most prevalent problems reported include depression, stress and anxiety for patients. For these patients, the fear of death, disease reoccurrence and distorted physical appearance may heighten the level of stress and unhappiness in the lives of patients, deteriorating their ability to manage. Chemotherapeutic regimens will temporarily paralyze the immune system, indicative by viewing the WBC and RBC counts, as well as cytokine levels of cancer patients. Other common effects as a result of receiving chemotherapies include toxicity to the gastrointestinal tract, myelosuppression and oral ulcers (14-17). Patients also often complain of deteriorated short-term memory.

The benefits of Tai Chi have been investigated in cancer patients who have been treated with chemotherapy for its psychological and biological effect. A cancer research also showed that tens of thousands of cancer patients are practicing TCC, which is being described as a mind-body practice to help deal with their disease (18) . And no wonder, scientists have found that TCC helps patients sleep better and cope with the pain, anxiety and depression often associated with traditional cancer treatments $(19,20)$. In addition, TCC may result in stimulation of the immune system. For example, Yeh, S. H et al observed (21) that the ratio of T helper cells (TH) to suppressor cells increased in Tai Chi practitioners. This study suggested that Tai Chi might be helpful to ameliorate diseases that involve immune suppression such as acquired immune deficiency syndrome and certain cancers treated with chemotherapy. Cancer patients who received chemotherapeutic agents represent a population with immune disorders. It has been reported that TCC practices can subtly enhance a cancer patient's immune system (22). Therefore, TCC may provide benefits to cancer patients who represent a population with multiple needs related to psychological stress and biological impacts.

To further understand the influence of practicing TCC on cancer patients, this study was designed to investigate the functions of TCC exercises to biological (WBC, RBC, cytokines, mucositis), psychological influence (state of happiness, level of stress, insomnia, appetite and short term memory), and forearm strength of patients, who were diagnosed with advanced invasive ductal carcinoma and had all received a mastectomy and combination chemotherapy. 


\section{Material and methods}

\subsection{Patients' selection}

In order to study the effects of regularly practicing TCC, a total of 60 subjects were recruited to this TCC study that took place from 2017 to 2020. Sixty female subjects, aged 32-42, of the patient group were recruited from West China Hospital of Stomatology, Sichuan University with a mean age of 37 (Table 1). The control group composed of 50 diseasefree, healthy female subjects, aged 26-36 (mean age of 31), were recruited as a comparison from the community. All subjects gave consent prior to enrollment, and the study protocol (2010SZ0065) was approved by the ethics committee of Sichuan University. The breast cancer patients had previously undergone a modified mastectomy for invasive ductal carcinoma (Table 2), and completed 4 cycles of FEC (500mg/m2 5-Fluorouracil, $75 \mathrm{mg} / \mathrm{m} 2$ Epirubicin and 500mg/m2 Cyclophosphamide) chemotherapy with a 3-week interval between each cycle.

Table 1 Physical characteristics of participants

\begin{tabular}{|l|l|c|l|c|}
\hline Parameters & $\begin{array}{l}\text { Healthy } \\
\text { group } \\
\text { (N=50) }\end{array}$ & $\begin{array}{l}\text { Baseline - Before } \\
\text { practicing Tai Chi Chuan } \\
\text { (N=60) }\end{array}$ & $\begin{array}{l}\text { 6 months after } \\
\text { Practicing Tai Chi Chuan } \\
\text { (N=60) }\end{array}$ & $\begin{array}{l}\text { 12 months after } \\
\text { practicing Tai Chi } \\
\text { Chuan (N=60) }\end{array}$ \\
\hline $\begin{array}{l}\text { Mean age } \\
\text { (years) }\end{array}$ & $31 \pm 5$ & $37 \pm 5$ & -- & $*-$ \\
\hline $\begin{array}{l}\text { Body weight } \\
\text { (kg) }\end{array}$ & $* 56.1 \pm 7.2$ & $49.2 \pm 6.5$ & $* 52.0 \pm 2.5$ & $* 53.5 \pm 1.4$ \\
\hline Body Height (m) & $1.5 \pm 1.2$ & $1.5 \pm 1.2$ & --- & - \\
\hline
\end{tabular}

*Statistically significant results $(\mathrm{p}<0.05)$ in comparison to the patient group at baseline; $( \pm)$ : Data were presented as mean \pm Standard Deviation.

Table 2 Characteristics of breast cancer patients $(\mathrm{N}=60)$

\begin{tabular}{|l|c|}
\hline Clinical parameters & Parameters \\
\hline Estrogen Receptor status $\left(*_{+}+\&_{+++}\right)$ & $80 \%$ \\
\hline Progesterone Receptor status $\left({ }^{+}+\&+++\right)$ & $78 \%$ \\
\hline HER-2/neu status( $\left.*_{+++}\right)$ & $41 \%$ \\
\hline Tumor Grading 1 \&2 & $61 \%$ \\
\hline Tumor Grading 3 & $39 \%$ \\
\hline Pre-menopause status & $98 \%$ \\
\hline Post-menopause status & $1 \%$ \\
\hline Lymphovascular permeation status & $34 \%$ \\
\hline${ }_{+++}^{*}+++:$ intensity of immunostaining in terms of protein expression
\end{tabular}

\subsection{Tai Chi Chuan classes}

The breast cancer survivors were allocated into the TCC group and taught the 18-form of Yang Style Tai Chi Chuan by the investigator (an experienced TC master with more than 15 years of practice and teaching experience). The 18-form is the basis of the traditional 115-form. Beginners will usually require 3-6 months of practice to be well acquainted with TCC, and similarly for these rehabilitating females. The Yang Style TCC routine was designed to constitute the representative components of the conventional schools by retraining the trunk, upper limbs and some of the lower limbs movements. During TCC training, accurate joint position and movement directions are encouraged. Weight shifting in different positions, as well as alternative thigh lifts was performed in a smooth and coordinated manner. Coordination between eye-hand, limbs-trunk were reinforced during TCC practice. Each class began with 5 minutes of warm-up, followed by the 18-style TCC practice, and ended with cool down. In total, the duration of the class was 50 minutes with breaks as deemed necessary. The investigator-cum-Tai Chi master led and monitored all classes. 


\subsection{Measurement of complete $\mathrm{WBC}, \mathrm{RBC}$ and serum cytokine levels}

Peripheral blood was collected from breast cancer patients at three intervals during the study after completion of chemotherapy treatment: prior to the start of the TCC exercise routine program (baseline), and at 6 and 12 months into the program. Blood from healthy subjects was drawn only once for data comparison.

Peripheral blood samples were collected by direct venipuncture from the arm vein of each subject. $3 \mathrm{ml}$ of blood was collected in EDTA tubes for measuring the total white blood cells (WBC), total red blood cells (RBC), while 6 ml was collected in clot blood tubes (BD Vacutainer, NJ, USA) for serum extraction to measure the level of cytokines by Enzymelinked immunosorbent assay (ELISA) analysis.

The serum level of cytokines IL2, IL4, IL6, IFN- $\gamma$ and TNF- $\alpha$ were measured by the ELISA kit (Diaclone, France; and Thermo Scientific, USA) at baseline and at 6 and 12 months after practicing TCC. For the serum extract from patients' blood, $50 \mu$ of the serum was pipetted into a 96-well plate, then incubated for 75 minutes at 750 rpm and washed with washing buffer three times. The wells were dried and read at $450 \mathrm{~nm}$ wavelength using Universal Microplate Reader (Sunrise, Tecan Co., Grödingen, Austria). The levels of cytokines in the samples were obtained by comparison with the standard curve generated from standards supplied by the manufacture. Each sample was analyzed in triplicates.

\subsection{Recording of oral ulcers}

The number of oral ulcers that developed during the course of treatment with FEC chemotherapy was recorded for all subjects during treatment and post-treatment, as well as at baseline, at 6 and 12 months into practicing TCC. The mean area of each ulcer was measured, and the number of days required for recovery from oral ulcers was also recorded. The intensity of pain as a result of development of the ulcer, measured by a scale of 1-10 was noted.

\subsection{Test of hand grip strength}

Hand grip strength can be measured quantitatively using an electronic hand dynamometer. The CAMRY EH101 (Guangdong, China) was used in this study to measure the strength of the female's hand grip strength. In a standing position, with the hand by the side at a neutral position (with elbow joint angle at 180 degrees), each participant was asked to hold the apparatus with the most force (for approximately 10 seconds). A recording was taken for both arms, and the side in which surgery was performed was noted. A measurement of the grip strength was recorded at baseline, 6 and at 12 months of TCC practice for each female. The device gives a precise digital reading of one's grip power in kilograms.

\subsection{Observation of physical performance}

All patients had their physical characteristics, including age, body weight, and height recorded at baseline, and at 6 and 12 months into practicing TCC (Table 1). Body weight was an imperative aspect of the study as the parameter was an indication of the influences of practicing TCC to the condition of body.

\subsection{Self-reported questionnaire}

A study questionnaire was conducted for each subject of the patient group at baseline, and at 6 and 12 months into the TCC program to investigate psychological parameters such as degree of happiness, stress, satisfaction of life, insomnia, and appetite. The measured parameters were formed from the basis of two questionnaires from our previous studies, which were revised and combined together as one questionnaire and given to participants $(23,24)$.Preliminary raw data were scaled from 1 to 10, with 1 indicating the lowest state for each parameter and 10 indicating the best state accordingly. The results of the questionnaires conducted at the three intervals were collected, grouped, analyzed, and presented in Figure 2.

\subsection{Regularly scheduled Tai Chi Chuan exercise regimen}

TCC exercises are recommended for post-operative breast cancer patients to alleviate pain and manage clinical symptoms. TCC exercises involve fluid and slow movements, and shifts, pulls and long stretches that increase circulation from shoulders to the arms. These movements are not forced, and are guided by the natural rhythm of one's breathing. When taking a deep breath, the chest rises up, the lungs expand, leading to a natural rise of the shoulders and arms. In the exhale, the chest and lungs relax, leading to a natural fall of the arm from the shoulder level down toward the waist.

The patient group consisting of 60 patients was taught 18 representative moves of the 115 Yang style TCC exercises. The Yang Style TCC routine was designed to constitute the representative components of the conventional schools by retraining the trunk, upper limbs and some of the lower limbs movements. These 18 movements were selected together 
with a physiotherapist with ample training for rehabilitation of breast cancer patients following surgery. These movements are taught with the goals of increasing the blood flow and circulation to the surgical areas. All subjects of the patient group were required to follow each posture, movement and motion demonstrated by the Tai Chi practitioner, with overseeing by physiotherapists. As part of the study, the subjects were asked to practice TCC for 50 minutes a day, 3 times a week for a period of 12 months. Females practicing TCC were advised not to begin other exercise programs during the course of the study and not to change their level of physical activity (25). To ensure that the patients were performing the exercises correctly, Tai Chi practitioners and physiotherapists were present to lead the TCC routines and gave advice and provided help as needed.

\subsection{Statistical analysis}

All calculations were performed using SPSS version 16.0. The ANOVA test was applied to measure the psychological and biological parameters of the healthy group, patient group at 6 and 12 months in comparison to the patient group at baseline. A P-value of less than 0.05 was considered statistically significant.

\section{Results}

There were significant improvements observed in the blood counts, cytokine levels, time of ulcer healing, handgrip strength and psychological states of the breast cancer survivors who practiced TCC regularly (all p $<0.05)$. All 60 enrolled female breast cancer survivors completed the study. The females had an $80 \%$ TCC class attendance with $100 \%$ compliance.

Regular TCC practice raised the WBC and RBC counts in post-chemotherapy patient group significantly (Table 3) $(\mathrm{p}<0.05)$ at month 6 and 12 in comparison to baseline. Prior to TCC practice, the blood counts (WBC and RBC) of breast cancer patients were outside the normal range (2.14 \pm 1.12 and 3.17 \pm 0.91$)$. At 6 and 12 months after the regular TCC regimen, the WBC count of the patient group raised statistically significant in comparison to baseline, respectively $(6.75 \pm 1.25,7.55 \pm 1.25$ vs $2.14 \pm 1.12, \mathrm{p}<0.05)$. Similarly, the RBC counts at 6 and 12 months compared to baseline were statistically significant $(4.09 \pm 0.82,4.62 \pm 1.25$ vs $3.17 \pm 0.91, \mathrm{p}<0.05)$. However, the WBC and RBC counts of the patient group at 12 months of TCC practice was slightly lower when compared to the healthy group at $7.55 \pm 1.25$ vs $8.04 \pm 1.05$ and $4.62 \pm 1.25$ vs $4.99 \pm 0.87$, respectively. Statistically significant results were also observed for hemoglobin for the patient group at 6 and 12 months compared to baseline (11.7 \pm 3.1 , and $12.8 \pm 1.25$ vs $8.9 \pm 2.86)$. The comparison of the number of platelets was insignificant across all groups and intervals of practicing TCC.

Table 3 Complete blood count of healthy female subjects and cancer patients at baseline and at 6 and 12 months after they had practiced TCC

\begin{tabular}{|c|c|c|c|c|c|c|}
\hline Parameters & $\begin{array}{l}\text { Healthy } \\
\text { group } \\
(\mathrm{N}=50)\end{array}$ & $\begin{array}{l}\text { Baseline - Before } \\
\text { practicing } \\
\text { Tai Chi Chuan } \\
(\mathrm{N}=60)\end{array}$ & $\begin{array}{l}6 \text { months after } \\
\text { practicing } \\
\text { Tai Chi Chuan } \\
(\mathrm{N}=60)\end{array}$ & $\begin{array}{l}12 \text { months after } \\
\text { practicing } \\
\text { Tai Chi Chuan } \\
(\mathrm{N}=60)\end{array}$ & Unit & $\begin{array}{l}\text { Normal } \\
\text { range }\end{array}$ \\
\hline White blood cell & $* 8.04 \pm 1.05$ & $2.14 \pm 1.12$ & $* 6.75 \pm 1.25$ & $* 7.55 \pm 1.25$ & $\mathrm{x} 109 / \mathrm{L}$ & $4.00-11.00$ \\
\hline Red blood cell & $* 4.99 \pm 0.87$ & $3.17 \pm 0.91$ & $* 4.09 \pm 0.82$ & $* 4.62 \pm 1.25$ & $\mathrm{x} 1012 / \mathrm{L}$ & $3.80-5.80$ \\
\hline Haemoglobin & $* 13.3 \pm 5.6$ & $8.9 \pm 2.9$ & $* 11.7 \pm 3.1$ & $* 12.8 \pm 1.3$ & $\mathrm{~g} / \mathrm{dL}$ & $11.5-16.5$ \\
\hline Platelet & $252 \pm 32$ & $183 \pm 11$ & $189 \pm 16$ & $231 \pm 5$ & x109/L & $150-400$ \\
\hline
\end{tabular}

*Statistically significant results $(\mathrm{p}<0.05)$ in comparison to the patient group at baseline; $( \pm)$ : Data were presented as mean \pm Standard Deviation

There was a significant positive correlation between performing TCC exercises longer and an improved level of cytokines. Figure 1 summarized the relationship between TCC performance and cytokine levels. At baseline, the levels of IL2, IL4, IL6, IFN- $\gamma$, and TNF- $\alpha$ cytokines in serum samples of breast cancer patients were significantly lower compared with the healthy group ( $\mathrm{p}>0.05)$. The cytokine level of IL2 in breast cancer patients at baseline in comparison to the healthy group was statistically significant $(34.43 \pm 3.78$ vs $130.18 \pm 16.22, \mathrm{p}<0.05)$. IL2 exhibited observable statistically significant increases after 6 and 12 months of TCC exercises compared to baseline in the patient group: $67.34 \pm 7.75$ and $118.15 \pm 13.59$ vs $34.43 \pm 3.78, p<0.05$, respectively. The level of cytokine IL2 had risen tremendously at 6 and 12 months of TCC practice (Figure 1). 


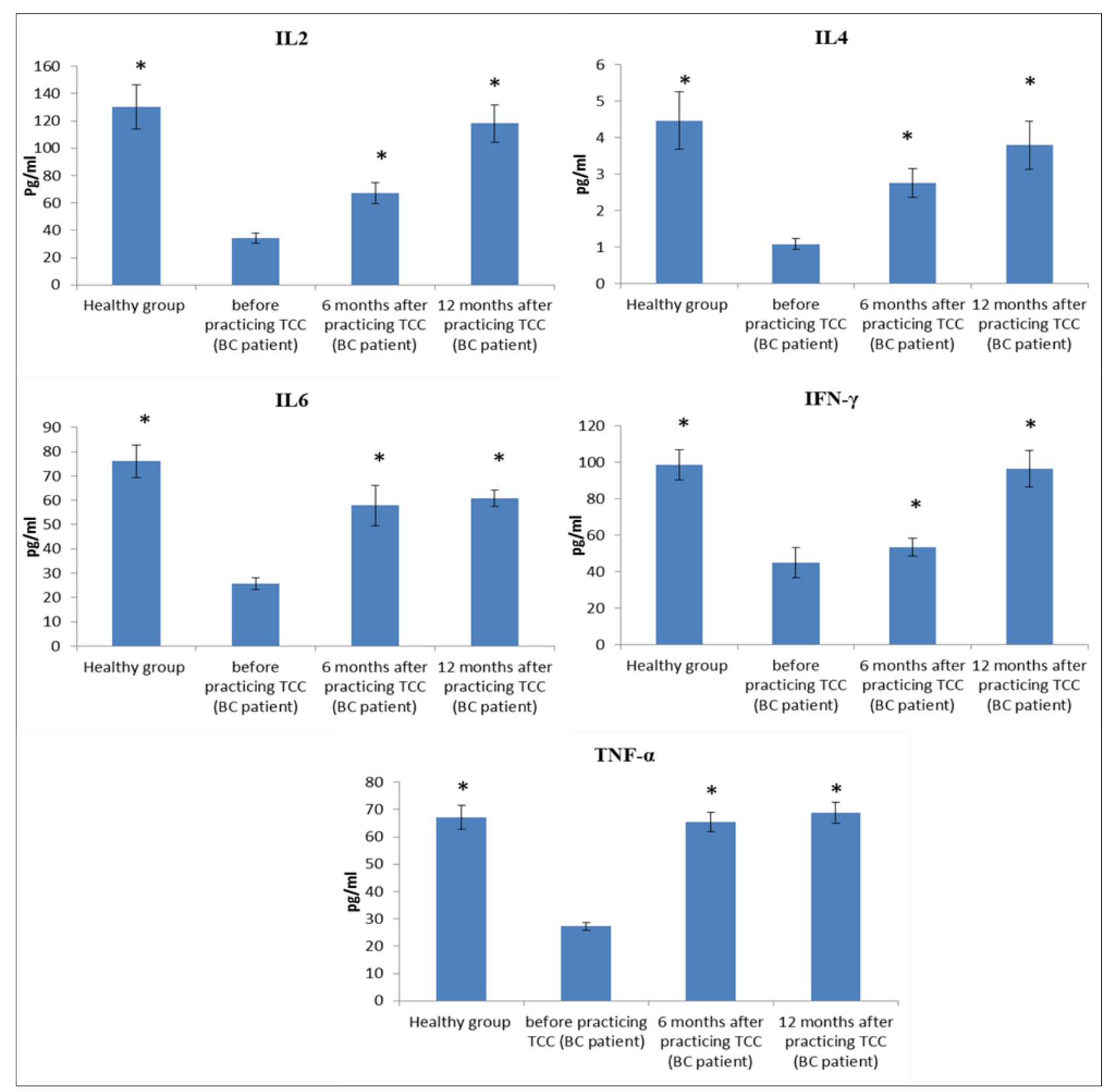

*Statistically significant results $(\mathrm{p}<0.05)$ in comparison to the patient group before practicing TCC. All data were presented as mean \pm Standard Deviation.

Figure 1 Different cytokines expression (IL2, IL4, IL6, IFN- $\gamma$ and TNF- $\alpha$ ) in the healthy group and patients group before practicing TCC (baseline) and at 6 and 12 months after practicing TCC were measured by the ELISA kit. Each sample was analysed in triplicates

The level of cytokine IL4 in the patient group at baseline compared to healthy controls was statistically significant $(1.08 \pm 0.15$ vs $4.47 \pm 0.78, \mathrm{p}<0.05)$. As shown in Figure 1, after 6 and 12 months of TCC exercises, the level of IL4 in patients was also noticeably higher than at baseline (2.76 \pm 0.39 and $3.80 \pm 0.66$ vs $1.08 \pm 0.15)$.

The levels of cytokines IL6, IFN- $\gamma$ and TNF- $\alpha$ in healthy controls were statistically significant in comparison to the patient group at baseline respectively: $76.09 \pm 6.73$ vs $25.76 \pm 2.39$; $98.61 \pm 8.45$ vs $44.94 \pm 8.41$; and $67.08 \pm 4.41$ vs $27.29 \pm 1.49$, all $\mathrm{p}<0.05$. The level of IL6 had risen significantly in the patient group after 6 and 12 months of regular TCC practice $(57.81 \pm 8.32$ and $60.87 \pm 3.39)$ compared to baseline $(25.76 \pm 2.39)$. Increase in the level of IFN- $\gamma$ was also observed in breast cancer patients after 6 and 12 months after the TCC program as opposed to baseline $(53.48 \pm 4.85$ and $96.34 \pm 9.96$ vs $44.94 \pm 8.41, \mathrm{p}<0.05$ ). Observable increase was also noted for TNF- $\alpha$ in the patient group after participation in the TCC regimen ( $65.45 \pm 3.58$ and $68.89 \pm 3.76$ vs $27.29 \pm 1.49)$. After 12 months of practicing TTC, there 
were no significant differences between the healthy controls and cancer patients who practiced TTC over 12 months $(\mathrm{p}>0.05)$.

Improved conditions of oral ulceration for patients were noted after implementation of the TCC regimen, albeit lack of significance. The patient group developed 6 oral ulcers $( \pm 1.0)$ during the 4-cycle chemotherapy treatment period and 3 oral ulcers $( \pm 1.0)$ in the post-chemotherapy-TCC practicing period. The mean area of the ulcer was $1.5 \mathrm{~mm}( \pm 0.3)$ in diameter during the TCC practicing period versus $3.75 \mathrm{~mm}( \pm 0.4)$ in chemo-drug treatment period. The degree of pain was rated at 3 during the TCC practicing period and 5 during the chemo-drug treatment period. The duration of the ulceration was 5-6 days in the TCC practicing period, opposed to 8-9 days in the chemotherapy treatment period.

A positive correlation was also noted between increased TCC practice, time, and hand grip strength with statistical significance (Table 4). At baseline, a comparison between healthy females and breast cancer cohorts showed the breast cancer females had significantly less hand grip strength ( $\mathrm{p}=0.000 ; 15.48 \mathrm{~kg}$ vs $23.31 \mathrm{~kg}$ ). At 6 months, the muscle strength of forearm in females practicing TCC was still lower than the healthy controls (17.19 kg vs $5.77 \mathrm{~kg})$ even though there was improvement when compared to the baseline. Following 12 months of TCC practice, breast cancer females demonstrated a stronger hand grip strength than baseline ( $\mathrm{p}=0.000,21.19 \mathrm{~kg}$ vs $15.48 \mathrm{~kg})$. However, no significant difference was noted between TCC-practicing survivors and healthy controls (21.19 kg vs $23.31 \mathrm{~kg}$; $\mathrm{p}=0.294$ ).

Table 4 Hand grip strength for healthy females at baseline, and for cancer patients at baseline, 6 and 12 months following TCC practice

\begin{tabular}{|c|c|c|c|c|c|c|}
\hline Parameter & $\begin{array}{l}\text { Healthy } \\
\text { group } \\
(\mathrm{N}=50)\end{array}$ & $\begin{array}{c}\text { Baseline Before } \\
\text { practicing Tai } \\
\text { Chi Chuan } \\
(\mathrm{N}=60)\end{array}$ & $\begin{array}{c}6 \text { months after } \\
\text { practicing } \\
\text { Tai Chi } \\
\text { Chuan }(\mathrm{N}=60)\end{array}$ & $\begin{array}{c}12 \text { months } \\
\text { after } \\
\text { practicing } \\
\text { Tai Chi } \\
\text { Chuan }(N=60)\end{array}$ & Unit & Normal range \\
\hline $\begin{array}{l}\text { Hand grip } \\
\text { strength }\end{array}$ & $23.3 \pm 6.5$ & $15.5 \pm 5.2^{* *}$ & $17.2 \pm 5.8^{* *}$ & $21.2 \pm 6.0^{\Delta \Delta}$ & $\mathrm{kg}$ & $\begin{array}{l}30-34: 21.5-35.3 \\
35-39: 20.3-34.1 \\
40-44: 18.9-32.7\end{array}$ \\
\hline
\end{tabular}
group at baseline.; $( \pm)$ : Data were presented as mean \pm Standard Deviation.

The results of the study questionnaire for measuring the psychological factors, including the state of happiness, level of stress, satisfaction of life, insomnia and appetite in cancer patients at baseline, and at 6 and 12 months into the regular TCC exercise routines were shown in Figure 2. The study questionnaire was conducted only once for the healthy subject group. At baseline, the state of happiness of patients diagnosed with breast cancer was significantly lower compared to the healthy subject group ( $5.60 \pm 0.82$ vs $7.20 \pm 0.54, \mathrm{P}<0.05)$. However, after participation in 12 month of regular TTC training, a higher degree of happiness was observed in patients in comparison to baseline $(8.20 \pm 0.52 \mathrm{vs} 5.60 \pm 0.82$, $\mathrm{P}<0.05)$, and no significant differences were observed when compared with the healthy controls. The degree of insomnia and appetite in patients before and after TCC training showed a similar pattern as the level of happiness. As shown in Figure 2, the degree of stress in patients before practicing TCC were significantly higher than the healthy group $(7.30 \pm 1.26$ vs $5.60 \pm 0.94, \mathrm{P}<0.05)$. Although the degree of stress decreased after 12 months of TCC exercise, patients still displayed a higher degree of stress than the healthy group. The appetite of breast cancer patients had also significantly

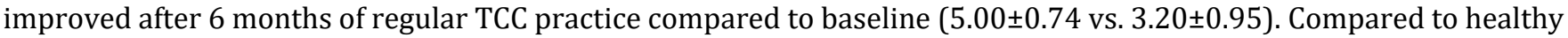
controls, however, the appetite of patients after practicing TCC for 12 months was still slightly lower at $7.90 \pm 0.77$ versus $6.90 \pm 0.73$, but it was statistically higher compared to baseline $(3.20 \pm 0.95)$. The basis of a good appetite was determined by the number of bowls of food consumed in every meal. If a patient had one bowl per meal or more, the patient was classified as having a good appetite. On the other hand, having less than one bowl of food was classified as having a bad appetite. 


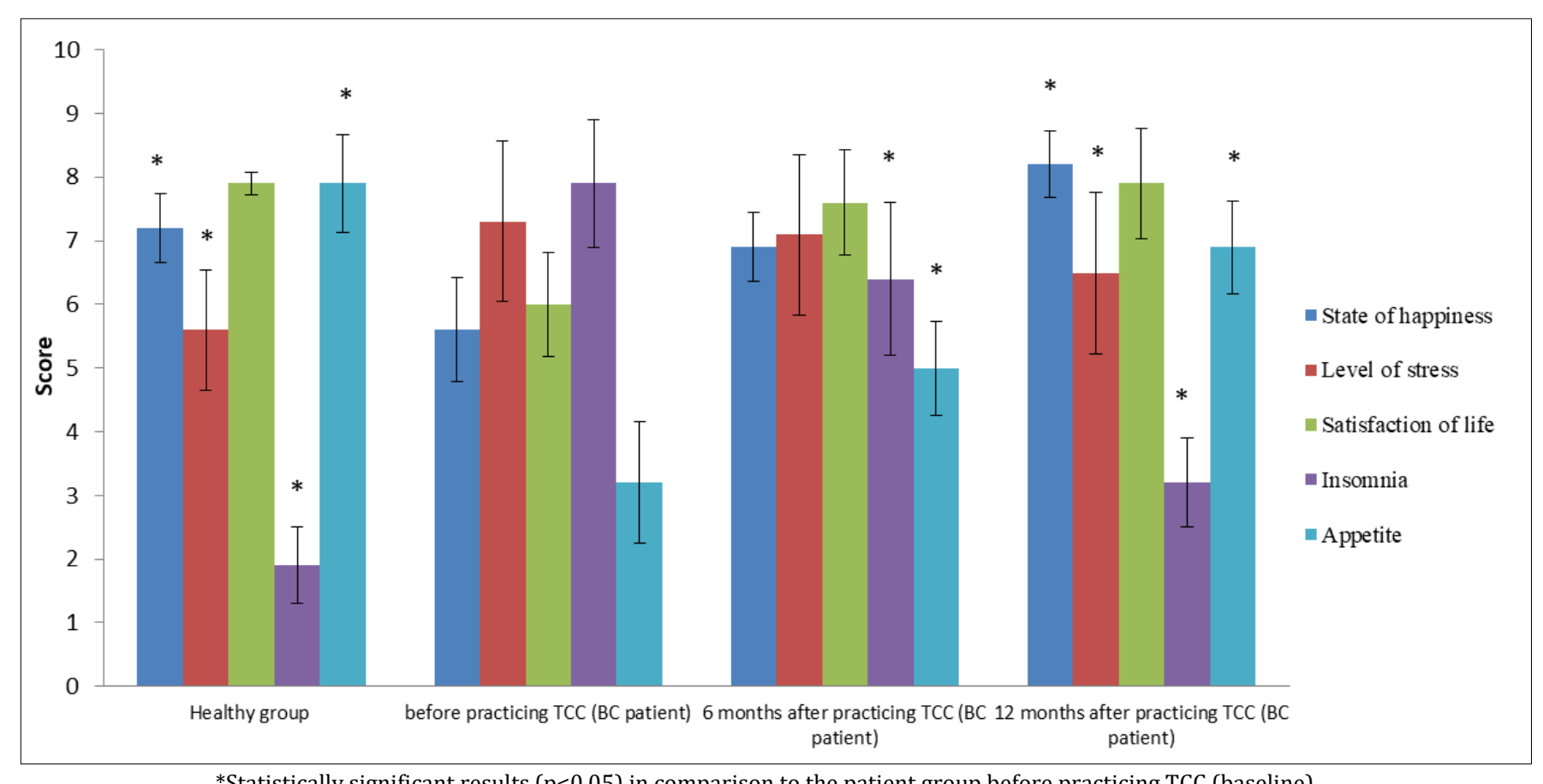

*Statistically significant results $(\mathrm{p}<0.05)$ in comparison to the patient group before practicing TCC (baseline). Data were presented as mean \pm Standard Deviation

Figure 2 Study questionnaire results of psychological factors between the groups

\section{Discussion}

TCC has long been a topic of interest for researchers and scientists owing to the positive benefits that accompany regular TCC practice for patients who suffer from various kinds of diseases, including breast cancer $(25,26)$. This study also demonstrated the importance of TCC for rehabilitation of breast cancer survivors. In addition to supporting the results of previous research, this study revealed that regular TCC practice will lead to biological, psychological and physical improvements and better overall health in the studied cohort. Practicing TCC helped breast patients to regain their appetite, which decreased as a result of chemotherapy. The average body weight of patients also increased as a result of performing TCC exercises. Psychological and psychosocial factors such as quality of life, stress, and insomnia also improved as reported by participants of this study who practiced TCC regularly for 12 months. To explain the outcomes observed, the mechanism by which of TCC directly improved the health of patients is elaborated below.

TCC is a low-impact, moderate intensity exercise that promotes relaxed breathing, flexibility, and mental concentration (27-29) . Throughout practicing TCC, the slow movement and continuity (29) gradually increases the body's metabolism (30) and blood circulation (31). As observed after 6 and 12 months of TCC practice, the RBC counts increased in patients, bringing the counts into the normal ranges. Improved RBC in cancer patients are essential and necessary for carrying oxygen and nutrients throughout the body, especially since the cells are eliminated by toxic chemotherapeutic regimens. Improved metabolism and blood circulation enhances the nutrients uptake and oxygen available for vital organs; hence, the poor appetite that resulted from chemotherapy was resolved and healthy cells and organs that were under attack was replenished and recovered. Eating more can improve one's health, raising a patient's immunity to diseases and providing more vital nutrients for cell production.

Chemotherapeutic agents work by eradicating abnormal growing cancer cells, as well as normal cells, thus directly suppressing the body's immune system (32). Low WBC count is normally observed in breast cancer patients receiving treatment as the immune system is under stress causing patients to be more prone to additional common illnesses (33, 34). Although exercise will without a doubt raise one's immunity and health, patients are often fatigued from treatment which renders such activity impossible $(35,36)$. Performing the 18-form TCC routine allowed the breast cancer patients of this trial to exercise without overworking their limited energy, which in turn allowed their bodies' immunity and defense mechanism to rebuild. As exemplified in this study, performing TCC following the completion of chemotherapy treatment allowed the WBC counts of patients to increase The WBC count in the patient subject group rose significantly at 6 and 12 months following the TCC program compared to baseline, which were outside the normal range. 
Practicing TCC post chemotherapy enhanced the production of cytokines (IL2, IL4, IL6, IFN- $\gamma$ and TNF- $\alpha$ ) significantly for subjects at 6 and 12 months after practicing TCC, compared to baseline that are important for cell signaling. Decreased levels of cytokines is another biomarker of a vulnerable immune system (37) . For the breast cancer female cohort under study, the levels of cytokines increased significantly at 6 and 12 months of TCC exercises compared to baseline. In parallel, the total WBC and RBC count also increased, indicating a stronger defense mechanism of the immune system in the patient group. A comparison of the levels of cytokines between the healthy group and patient group at 12 months of TCC exercise showed similar results, all of which were within the normal range. As patients practicing TCC have shown an increase in total WBC and RBC counts and the levels of serum cytokines (IL2, IL4, IL6, TNF- $\alpha$ and IFN- $\gamma$ ), it is recommended for breast cancer patients to practice TCC to reinforcing the immune system due to the suppression of estrogen and WBC levels.

For the participants who regularly practiced TCC, improved muscle strength of the hand grip strength was noted in this study. In line with previous pilot studies, we demonstrated that long-term TCC exercises could lead to improved muscle strength $(25,38)$. Although TCC is a low impact exercise, the emphasis is on controlled, smooth movements of the muscles of upper limbs, training the arms to move with more force and power. A longer duration of TCC practice correlated with improved forearm strength as measured by the handgrip dynamometer $(25,38)$. From a rehabilitation standpoint, avoidance of overexertion of the arm is recommended following surgery. However, following adequate rest, it is necessary to strengthen the limbs by performing stretches and exercises as recommended by a physiotherapist. The positive correlation between TCC exercises and improved forearm strength, therefore, could be explained by the high attendance rates of the participants and their continued practice of TCC exercises (25) .

Oral ulcerative mucositis, another complication of cytotoxic chemotherapy, is associated with significant morbidity, pain, odynodysphagia, dysgeusia, and subsequent dehydration and malnutrition that all work to reduce the quality of life of affected patients (39-41) . Approximately 20\%-40\% of patients who received chemotherapy for the treatment of cancer also developed ulcerative lesions $(40,42)$. Practicing TCC may have the potential to improve the immunity of cancer patients through increasing the blood circulation as indicated by RBC counts and bone marrow production of the immune cells (30). In addition, regular practice of TCC may help to improve the healing of wounds and reinforce the oral mucosa to combat the toxicity of chemotherapy. The results of this study demonstrated that practicing TCC could boost the immune system for immuno-suppressive patients and improve their quality of life.

Social support is likely to influence perceived self-efficacy and thus TCC exercise behaviour $(43,44)$. Participating in group practice of TCC provided ample chances for participants to make new friends and share their difficulties. Psychological factors, including improvements in happiness, stress, satisfaction of life, insomnia and appetite (45) was recorded for patients who practiced TCC in the current study (Figure 2). As mind concentration is highly emphasized, patients who practiced TCC reported that they felt calm, relaxed, with reduced pain and could temporarily be freed from social pressures $(46,47)$.

The state of happiness of the patient group was reportedly higher at 12 months in comparison to the baseline results. This meditation practice could internally affect the secretion of hormone levels. Stress hormone secretions had reduced after practicing TCC compared to baseline, which was indicated by the results of this study. However, the degree of stress in patients after TCC exercise was still be considerably higher in comparison to healthy subjects. Cancer-related stress remained higher in cancer patients, because there is still the fear of death and uncertainty of disease progression or recurrence of disease. Although the level of stress was noticeably lower in cancer patients at 6 and 12 months of regular TCC practice, the level of stress in patients may need to be verified post-study.

TCC is a fluid movement exercise that increases the metabolism of the body (47) . Nutrients absorption and waste excretions are facilitated; patients' appetite (48) was also noticeably better after practicing TCC. Regular exercise induces tiredness, allowing people to fall asleep more easily at night (49). Indeed, lessened insomnia was confirmed in the patient group after practicing TCC. Improvements to short term memory were also noted by patients of the study group, who complained of poor memory during chemotherapy treatment $(24,50,51)$. Patients noted that practicing TCC allowed their minds to be more clear, focused and attentive, thus allowing them to better concentrate on small details and improving their memory $(24,50,51)$.

\section{Limitation}

In the present study, there was a lack of control group and the question of natural recovery of the breast cancer survivors cannot be answered. The improvement of the health status as reflected by the biological markers and psychological perspectives might be due to exercise, not necessarily TCC per se. Moreover, this study was prospective in nature and the non-randomization of participants may lead to the existence of confounding placebo factor. Nevertheless, a 
randomized clinical trial with 3 arms, namely TCC, physiotherapeutic exercise and control groups are being undertaken by the same research group to address the aforementioned limitations.

\section{Conclusion}

In summary, regular practice of TCC is highly recommended for patients who received mastectomy and chemotherapy after being diagnosed with breast cancer. Through practicing TCC, patients made more friends and were able to expand their social network, which might help them to fight against the disease. Improvements of biological and psychological factors have been shown for patients who regularly practiced TCC in this study. Biological factors, such as WBC and RBC count, levels of cytokines (IL2, IL4, IL6, IFN- $\gamma$, TNF- $\alpha$ ) and oral ulcers all improved after practicing TCC. Improved hand grip strength was noted for the participants who had surgery performed for their breast cancer. For psychological factors, patients reported improvements in happiness, quality of life, insomnia, and improved appetite. After 12 months of regular TCC exercise, improved short term memory was also noted by the patient group, whom all felt was hindered under the influence of chemotherapeutic treatment. However, the effects of regular TCC regimen on short term memory in combination with improved psychological and biological influences for post-chemotherapy breast cancer patients warrant further evaluation.

\section{Compliance with ethical standards}

\section{Acknowledgments}

The authors would like to thank Professor Liang Hao of Department of Prosthodontics West China College of Stomatology, State Key Laboratory of Oral Diseases \& National Clinical Research Center for Oral Diseases, Sichuan University for recruiting breast cancer survivors and assisting management TCC class.

\section{Disclosure of conflict of interest}

We declare that we have no financial and personal relationships with other people or organizations that can inappropriately influence our work, there is no professional or other personal interest of any nature or kind in any product, service and/or company that could be construed as influencing the position presented in, the article entitled, "Tai Chi Chuan improves cytokine expression levels and quality of life in post chemotherapy breast cancer survivors".

\section{Statement of informed consent}

Informed consent was obtained from all individual participants included in the study.

\section{References}

[1] Adler PA, Roberts BL. The use of Tai Chi to improve health in older adults. Orthop Nurs. 2006; 25(2): $122-126$.

[2] Boedicker F, Boedicker M. The Philosophy of Tai Chi Chuan: Wisdom from Confucius, Lao Tzu, and Other Great Thinkers. 2011.

[3] Thornton EW, Sykes KS, Tang WK. Health benefits of Tai Chi exercise: improved balance and blood pressure in middle-aged women. Health Promot Int. 2004; 19(1): 33-38.

[4] Han A, Robinson V, Judd M, et al. Tai chi for treating rheumatoid arthritis. Cochrane Database Syst Rev. 2004 ; 3: 0.

[5] Zhou D-h. Comparative Therapeutic Exercise: East and West. Am J Chin Med. 1978; 6(04): 263-271.

[6] Yeh GY, Wang C, Wayne PM, Phillips RS. The effect of tai chi exercise on blood pressure: a systematic review. Prev Cardiol. 2008; 11(2): 82-89.

[7] Bratun U, Asaba E. From individual to communal experiences of occupation: Drawing upon Qi Gong practices. J Occup Sci. 2008; 15(2): 80-86.

[8] Sandlund ES, Norlander T. The effects of tai chi chuan relaxation and exercise on stress responses and well-being: an overview of research. Int J Stress Manag. 2000; 7(2): 139-149.

[9] Taylor-Piliae RE, Haskell WL, Waters CM, Froelicher ES. Change in perceived psychosocial status following a 12week Tai Chi exercise programme. J Adv Nurs. 2006; 54(3): 313-329. 
[10] Köstler WJ, Hejna M, Wenzel C, Zielinski CC. Oral mucositis complicating chemotherapy and/or radiotherapy: options for prevention and treatment. CA: a cancer journal for clinicians. 2001; 51(5): 290-315.

[11] Sonis S, Sonis A, Lieberman A. Oral complications in patients receiving treatment for malignancies other than of the head and neck. J Am Dent Assoc. 1978; 97(3): 468-472.

[12] Dreizen S, McCredie K, Keating M. Chemotherapy-induced oral mucositis in adult leukemia. Postgrad Med. 1981; 69(2): 103.

[13] Sonis S, Clark J. Prevention and management of oral mucositis induced by antineoplastic therapy. Oncology (Williston Park, NY). 1991; 5(12): 11.

[14] van der Wall E, Rutgers EJ, Holtkamp MJ, et al. Efficacy of up-front 5-fluorouracil-epidoxorubicincyclophosphamide (FEC) chemotherapy with an increased dose of epidoxorubicin in high-risk breast cancer patients. Br J Cancer. 1996; 73(9): 1080-1085.

[15] Poole CJ, Earl HM, Hiller L, et al. Epirubicin and cyclophosphamide, methotrexate, and fluorouracil as adjuvant therapy for early breast cancer. N Engl J Med. 2006; 355(18): 1851-1862.

[16] Fornier M ,Norton L. Dose-dense adjuvant chemotherapy for primary breast cancer.Breast Cancer Res. 2005; 7(2): 64-69.

[17] Janni W, Rjosk D, Strobl B, et al. Chemotherapy-associated myelosuppression in gynecological oncology. Gynakol Geburtshilfliche Rundsch. 2001; 41(3): 166-173.

[18] Mansky P, Sannes T, Wallerstedt D, et al. Tai chi chuan: mind-body practice or exercise intervention? Studying the benefit for cancer survivors. Integr Cancer Ther. 2006; 5(3): 192-201.

[19] Field T, Diego M, Delgado J, Medina L. Tai chi/yoga reduces prenatal depression, anxiety and sleep disturbances. Complement Ther Clin Pract. 2013; 19(1): 6-10.

[20] Nguyen MH, Kruse A. A randomized controlled trial of Tai chi for balance, sleep quality and cognitive performance in elderly Vietnamese. Clin Interv Aging. 2012; 7: 185-190.

[21] Yeh SH, Chuang H, Lin LW, Hsiao CY, Eng HL. Regular tai chi chuan exercise enhances functional mobility and CD4CD25 regulatory T cells. Br J Sports Med. 2006; 40(3): 239-243.

[22] Wang R, Liu J, Chen P, Yu D. Regular Tai Chi exercise decreases the percentage of type 2 cytokine-producing cells in postsurgical non-small cell lung cancer survivors. Cancer Nurs. 2012.

[23] Bai LJ, Liu Q, Wang M, et al. Evaluation of the psychological and biological changes of patients diagnosed with benign and malignant breast tumors. Int J Biol Markers. 2012; 27(4): e322-330.

[24] Loo WT, Yip MC, Chow LW, et al. A pilot study: application of hemoglobin and cortisol levels, and a memory test to evaluate the quality of life of breast cancer patients on chemotherapy. Int J Biol Markers. 2013; 28(4): e348356.

[25] Mustian KM, Palesh OG, Flecksteiner SA. Tai Chi Chuan for breast cancer survivors. Med Sport Sci. 2008; 52: $209-$ 217.

[26] Lee MS, Choi TY, Ernst E. Tai chi for breast cancer patients: a systematic review. Breast Cancer Res Treat. 2010; 120(2): 309-316.

[27] Luskin FM, Newell KA, Griffith M, et al. A review of mind/body therapies in the treatment of musculoskeletal disorders with implications for the elderly. Altern Ther Health Med. 2000; 6(2): 46-56.

[28] Ospina MB, Bond K, Karkhaneh M, et al. Meditation practices for health: state of the research. Evid Rep Technol Assess (Full Rep). 2007; (155): 1-263.

[29] Yang Y, Verkuilen JV, Rosengren KS, et al. Effect of combined Taiji and Qigong training on balance mechanisms: a randomized controlled trial of older adults. Med Sci Monit. 2007; 13(8): CR339-348.

[30] Li JX, Hong Y, Chan KM. Tai chi: physiological characteristics and beneficial effects on health. Br J Sports Med. 2001; 35(3): 148-156.

[31] Wang JS, Lan C, Wong MK. Tai Chi Chuan training to enhance microcirculatory function in healthy elderly men. Arch Phys Med Rehabil. 2001; 82(9): 1176-1180.

[32] Diwanay S, Gautam M, Patwardhan B. Cytoprotection and immunomodulation in cancer therapy. Curr Med Chem Anticancer Agents. 2004; 4(6): 479-490. 
[33] Vollhardt LT. Psychoneuroimmunology: A literature review. Am J Orthopsychiatry. 1991; 61(1): 35-47.

[34] Dale DC. Neutropenia and neutrophilia. Hematology. Macgraw-Hill, New York. 2001; 823-834.

[35] Lahans T. Integrating Chinese and conventional medicine in colorectal cancer treatment. Integr Cancer Ther. 2007; 6(1): 89-94.

[36] Bragg PC, Bragg P. Super Power Breathing. 1999: Health Science Publications, Inc.

[37] Gabriel H, Kindermann W. The acute immune response to exercise: what does it mean? Int J Sports Med. 1997; 18(1): S28.

[38] Mustian KM, Katula JA, Zhao H. A pilot study to assess the influence of tai chi chuan on functional capacity among breast cancer survivors. J Support Oncol. 2006; 4(3): 139-145.

[39] Kostler WJ, Hejna M, Wenzel C, Zielinski CC. Oral mucositis complicating chemotherapy and/or radiotherapy: options for prevention and treatment. CA Cancer J Clin. 2001; 51(5): 290-315.

[40] Dreizen S, McCredie KB, Keating MJ. Chemotherapy-induced oral mucositis in adult leukemia. Postgrad Med. 1981; 69(2): 103-108, 111-102.

[41] Bey A, Ahmed SS, Hussain B, Devi S, Hashmi SH. Prevention and management of antineoplastic therapy induced oral mucositis. Natl J Maxillofac Surg. 2010; 1(2): 127-134.

[42] Sonis S, Clark J. Prevention and management of oral mucositis induced by antineoplastic therapy. Oncology (Williston Park). 1991; 5(12): 11-18. discussion 18-22.

[43] Warner LM, Ziegelmann JP, Schuz B, Wurm S, Schwarzer R. Synergistic effect of social support and self-efficacy on physical exercise in older adults. J Aging Phys Act. 2011; 19(3): 249-261.

[44] McAuley E, Jerome GJ, Marquez DX, Elavsky S, Blissmer B. Exercise self-efficacy in older adults: social, affective, and behavioral influences. Ann Behav Med. 2003; 25(1): 1-7.

[45] Kim J. Perception of Social Change and Psychological Well-Being: A Study Focusing on Social Change in Korea Between 1997 and 20001. J Appl Soc Psychol. 2008; 38(11): 2821-2858.

[46] Goodill SW. An introduction to medical dance/movement therapy: Health care in motion. 2005: Jessica Kingsley Publishers.

[47] Smith JC. Relaxation, meditation, \& mindfulness: A mental health practitioner's guide to new and traditional approaches. 2005.

[48] Sharma SK. Alternate Therapies. 2000: Diamond Pocket Books (P) Ltd.

[49] MEISLER JG. Toward optimal health: the experts respond to sleep deprivation. J Womens Health. 1998; 7(10): 1205-1210.

[50] Bower JE. Behavioral symptoms in patients with breast cancer and survivors. J Clin Oncol. 2008; 26(5): 768-777.

[51] Cella D, Fallowfield LJ. Recognition and management of treatment-related side effects for breast cancer patients receiving adjuvant endocrine therapy. Breast Cancer Res Treat. 2008; 107(2): 167-180. 